Sich einen Namen Machen 
Ergebnisse der Frauenforschung

Band 41

Begründet und im Auftrag des Präsidenten der Freien Universität Berlin herausgegeben von

Prof. Anke Bennholdt-Thomsen, Germanistik

Elisabeth Böhmer, Soziologie

Prof. Marlis Dürkop, Sozialpädagogik

Prof. Ingeborg Falck, Medizin

Prof. Marion Klewitz, Geschichtsdidaktik

Prof. Jutta Limbach, Jura

Prof. Hans Oswald, Pädagogik

Prof. Renate Rott, Soziologie

Dr. Hanna Beate Schöpp-Schilling, Amerikanistik/Anglistik, Germanistik

Prof. Margarete Zimmermann, Romanistik

Koordination: Anita Runge 
Susanne Kord

\section{SICH EINEN NAMEN}

MACHEN

Anonymität und weibliche Autorschaft 1700-1900

Verlag J. B. Metzler

Stuttgart · Weimar 
Die Deutsche Bibliothek - CIP-Einheitsaufnahme

Kord, Susanne:

Sich einen Namen machen : Anonymität und weibliche Autorschaft 1700 - 1900 / Susanne Kord. Stuttgart ; Weimar : Metzler, 1996

(Ergebnisse der Frauenforschung; Bd. 41)

ISBN 978-3-476-01438-2

ISBN 978-3-476-03648-3 (eBook)

DOI $10.1007 / 978-3-476-03648-3$

NE: GT

Dieses Werk einschließlich aller seiner Teile ist urheberrechtlich geschützt. Jede Verwertung außerhalb der engen Grenzen des Urheberrechtsgesetzes ist ohne Zustimmung des Verlages unzulässig und strafbar. Das gilt insbesondere für Vervielfältigungen, Übersetzungen, Mikroverfilmungen und die Einspeicherung und Verarbeitung in elektronischen Systemen.

(C) 1996 Springer-Verlag GmbH Deutschland

Ursprünglich erschienen bei J. B. Metzlersche Verlagsbuchhandlung

und Carl Ernst Poeschel Verlag GmbH in Stuttgart 1996 
Für meine Familie:

Magdalena Pfannkuchen, 1921-1973

Hedwig Pfannkuchen, ${ }^{*} 1914$

Julie Pfannkuchen, 1890-1977

Eva Gobiet, *1926

Susan Cocalis, *1947

Ihnen verdanke ich

die Liebe zum Lesen

die Voraussetzung zum Schreiben

den Mut zum Veröffentlichen 
„Nomen est omen.“

Alte römische Weisheit

„O, be some other name!

What's in a name?"

William Shakespeare, Romeo and Juliet

„Name ist Schall und Rauch.“

Johann Wolfgang Goethe, Faust

"Genius of a sort must have existed among them [...], but certainly it never got itself onto paper. When, however, one reads of a woman possessed by the devils, of a wise woman selling herbs, or even a remarkable man who had a remarkable mother, then I think we are on the track of a lost novelist, a suppressed poet, or some Emily Brontë, who dashed her brains out on the moor, crazed with the torture her gift had put her to."

Virginia Woolf, A Room of One's Own

„For most of history, Anonymous was a woman.“

Virginia Woolf 


\section{INHALTSVERZEICHNIS}



I. Sich einen Namen machen: Zur Anonymität und Pseudonymität weiblicher Autoren zwischen 1700 und 1900 . . . . . . . . . . 11

1. Die Frau, das unbekannte Wesen: „Jemand“ und "Tantchen Ungenannt" . . . . . . . . . . . . . . . . . . . . . 11

2. Die Frau als Opfer und Komplizin: Zum literarischen Ausschluß von Frauen . . . . . . . . . . . . . . 20

3. „Ain't I a Woman?“ oder Kleider machen Frauen: Methodologische Überlegungen zur Anonymität und Identität der Frau . . . . . . . . . 27

II. Der kurze Weg zur Männlichkeit: Von Jenny (1753-1807) zu Paul Dido (1826-1891) . . . . . . . . 36

1. Das neue Rollenangebot: Männliche Modelle zur ,Natur und ,Bestimmung der Frau . . . . . . . . . . . . . 36

2. Frauenrollen außer Haus: Weiblichkeit auf dem Theater . . . . . . . 44

3. Und drinnen waltet die züchtige Hausfrau: Frauen spielen ihre, Weiblichkeit ${ }^{t} \ldots \ldots$. . . . . . . . . 46

4. Der kurze Weg zur Männlichkeit: Weibliche und männliche Pseudonyme . . . . . . . . . . 51

III. Anonymität und das Genre als Geschlecht . . . . . . . . . . . 56

1. Genre, Genie und Geschlecht . . . . . . . . . . . . . 56

2. Genre und weibliche Anonymität: Dramen und Romane von Frauen . . . . . . . . . . . . . . 67

3. Die Schaubühne als unmoralische Anstalt betrachtet: Zum Unterschied zwischen Drama und Theater . . . . . . . . . . 69

4. Das ,andere' Genre: Theater als, weiblich' . . . . . . . . . . . 70

5. Dramen und Theaterstücke von Frauen . . . . . . . . . . . . 74

IV. Das Land der Dichterinnen und Denkerinnen: Klassenspezifische Namenlosigkeit . . . . . . . . . . . . . 77

1. Das Bürgertum als Kultur und die Ausgrenzung von Frauen . . . . . 77

2. Zur Rhetorik in Schriften nicht/bürgerlicher Autorinnen: Dilettantismus und Klassenkampf . . . . . . . . . . . . . . 85

3. Anonymität und Klasse: Beispiele aus dem 18. Jahrhundert . . . . . 90 
V. In eigener Sache: Frauen über ihre Anonymität und Autorschaft . . . 93

1. „Zwei Seelen, wohnen, ach, in meiner Brust“:

Schuld und Schizophrenie weiblicher Autorschaft . . . . . . . . . . 98

2. „Gleich groß und verehrungswürdig als Frau wie als Dichterin“:

Anmerkungen zu einem unerreichbaren Ziel . . . . . . . . . 112

3. Die Freiheit, die sie meinten?

Anonymität und die Utopie der Geschlechtslosigkeit . . . . . . . . 122

VI. Die prätendierte Weiblichkeit: Der Autor als Autorin . . . . . . 125

VII. Selbstgewählte versus effektive Anonymität:

Zur Rezeptionsgeschichte weiblicher Autoren . . . . . . . . . . 135

1. Das Integrationsmodell:

Frauen in männlicher Literaturgeschichtsschreibung. . . . . . . . 136

2. „Sie war kein Genie“:

Musen, Wahnsinnige und andere Ausnahmen _ . . . . . . . . 140

3. Biographismus in der Kritik: Zwei Fallstudien. . . . . . . . . . . 147

4. Das Geschlecht in der Kritik:

,Männliche' und ,weibliche' Naturen . . . . . . . . . . . . . 156

5. Eine Literaturgeschichte für sich allein:

Aufarbeitung, Ghettoisierung, Ideologisierung, Theoretisierung. . . . 164

VIII. Schlußbemerkung und zwei Denkanstöße . . . . . . . . . . 174

1. Kein Zimmer für sich allein: Voraussetzungen und Produktionsbedingungen weiblicher Autorschaft . . . . . . . . 175

2. Anonymität oder Trivialität:

Zu den Alternativen weiblicher Autorschaft . . . . . . . . . . 178

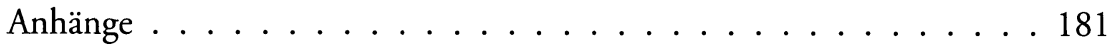

A. Erwähnte Schriftstellerinnen und Pseudonyme . . . . . . . . . . . 181

B. Ermittelte Pseudogynyme . . . . . . . . . . . . . . . . . . . . . . 198

C. Relevante Termini . . . . . . . . . . . . . . . . . . . . 200

Literaturverzeichnis . . . . . . . . . . . . . 202 


\section{Vorwort}

Allen meinen Freunden und Freundinnen, davon vielen ohne akademische Ausbildung, bin ich wie immer dankbar für ihre Unterstützung, ihre Geduld und ihr scheinbar unbegrenztes Interesse an meiner Arbeit. Das gilt besonders für meinen besten Freund, John Landau, der an diesem Projekt intensiv beteiligt war - von der ersten Idee vor vier Jahren bis zum Schlußpunkt vor wenigen Wochen. Außerdem danke ich

Friederike Eigler, die das dritte Kapitel las und freundlich, aber kritisch beurteilte,

Anita Runge und Helga Brandes für Literaturhinweise,

der Georgetown University für die Stipendien im Sommer und Herbst 1994, die mir die Fertigstellung des Buches früher ermöglichten als gedacht, und der University of Nebraska Press für ihre freundliche Erlaubnis zur Übersetzung einiger Passagen und zur Wiederverwendung einiger Ideen und Zitate aus meinem Aufsatz „Und drinnen waltet die züchtige Hausfrau? Caroline Pichler's Fictional Auto/Biographies" im fünften und siebten Kapitel. Der besagte Aufsatz erschien ursprünglich im Women in German Yearbook 8 (1992): 141-58.

Zitate aus dem Englischen und Französischen sind übersetzt, die Originalzitate finden sich in Fußnoten. Wo nicht anders vermerkt, stammen alle Übersetzungen von mir.

Werke von Autorinnen des 18. und 19. Jahrhunderts erscheinen im Literaturverzeichnis unter genauer und ungekürzter Übernahme der bibliographischen Angaben in der benutzten Ausgabe, mit Ausnahme des Verlagsortes. (Beispiel: Delle Grazie, M[arie] E[ugenie]. Saul. Tragödie in fünf Acten von M. E. delle Grazie. Wien 1885.) Der Grund: oft finden sich schon im Titelblatt reichhaltige Aufschlüsse über die Spielarten der Anonymität, Teilanonymität, Geschlechtsanonymität oder auch betonter Orthonymität. Einige Autorinnen unterschlugen ihren Vornamen und damit ihr Geschlecht („M. Günther"), viele andere verweigerten ihren ganzen Namen, teilweise unter Vorspiegelung eines männlichen oder weiblichen Geschlechts (die vielen „Verfasser“ oder „Verfasserinnen“ eines anderen, im Titel genannten Werkes). Wieder andere entzogen sich - und ich meine, ausdrücklich - dieser weiblichen Tradition der Anonymität, indem sie sich im Titel überidentifizierten: $z$. B. durch zusätzliche Nennung des Geburtsnamens, so als befürchteten sie, mit einer gleichnamigen Autorin verwechselt zu werden („Caroline Pichler, geborne von Greiner“) oder durch die angesichts des Vornamens überflüssige Hervorhebung des Geschlechts („Frau Hedwig Henrich-Wilhelmi“, eine Angabe, mit der vielleicht 
auch der Status der Autorin als Ehefrau betont werden sollte). Angesichts des Themas habe ich für den Aussagewert dieser Titelblätter etwaige Wiederholungen und Titel von teilweise barocker Länge gern in Kauf genommen.

Ich habe mich beim Schreiben dieses Buches redlich bemüht, dem sogenannten ,generischen' Maskulinum (,der Leser', ,der Autor ' usw.) endlich den verdienten Abschied zu geben und zwischen Autorinnen (= nur Frauen), Autoren (= nur Männer) und Autorinnen und Autoren beiderlei Geschlechts zu unterscheiden. Von Autoren zu sprechen und damit angeblich Autorinnen ,mitzumeinen' kann nur, wer sich nie mit dem Thema Anonymität der Frau beschäftigt hat und außerdem auf stilistische Genauigkeit keinen großen Wert legt. Im allgemeinen sollten Leserinnen und Leser also davon ausgehen, daß mit Maskulina wie Autoren, Leser etc. tatsächlich ausschließlich Männer gemeint sind. Daß mir auch nach viermaligem Korrekturlesen Fehler in dieser Hinsicht unterlaufen sein könnten, halte ich durchaus für möglich: jahrzehntelange Gewohnheiten, auch falsche, lassen sich nicht in wenigen Monaten ablegen.

Auszüge aus dem dritten, vierten, fünften und siebten Kapitel gingen, meist in anderer Form, in verschiedene Vorträge ein: bei der Modern Language Association in Toronto, Ontario (Dezember 1993), an der Library of Congress in Washington, D. C. (Juli 1994), bei der German Studies Association in Dallas, Texas (September 1994), bei der Modern Language Association in San Diego, California (Dezember 1994), und an der Freien Universität Berlin (Dezember 1994). Mein Dank gilt den Teilnehmerinnen und Teilnehmern an diesen Symposien, deren Kommentare und Kritik meinen Blick für theoretische und methodologische Aspekte des Themas schärften und so die vorliegende Version mitermöglichten. 